\title{
SECOND-ORDER DIFFERENTIAL EQUATIONS WITH DEVIATING ARGUMENTS
}

\author{
T. JANKOWSKI AND W. SZATANIK
}

Received 2 May 2006; Revised 22 May 2006; Accepted 28 May 2006

This paper deals with boundary value problems for second-order differential equations with deviating arguments. Some sufficient conditions are formulated under which such problems have quasisolutions or a unique solution. A monotone iterative method is used. Examples with numerical results are added to illustrate the results obtained.

Copyright (c) 2006 T. Jankowski and W. Szatanik. This is an open access article distributed under the Creative Commons Attribution License, which permits unrestricted use, distribution, and reproduction in any medium, provided the original work is properly cited.

\section{Introduction}

Let us consider a problem

$$
\begin{aligned}
x^{\prime \prime}(t)=f(t, x(t), x(\alpha(t))) & \equiv F x(t), \quad t \in J=[0, T], T<\infty, \\
x(0)=0, \quad x(T) & =r x(\gamma) \quad \text { with } \gamma \in(0, T),
\end{aligned}
$$

where $f \in C(J \times \mathbb{R} \times \mathbb{R}, \mathbb{R})$ and $\alpha \in C(J, J)$ (e.g., $\alpha$ may be defined by $\alpha(t)=\sqrt{t}, T \geq 1$ or $\alpha(t)=0.7 t, t \in J)$. Moreover, $r$ and $\gamma$ are fixed real numbers.

Differential equations with deviated arguments arise in a variety of areas of biological, physical, and engineering applications, see, for example, [9, Chapter 2]. The monotone iterative method is useful to obtain approximate solutions of nonlinear differential equations, for details see, for example, [10], see also [1-8, 11, 12]. It has been applied successfully to obtain results of existence of quasisolutions for problems of type (1.1), see [4]. In paper [4], it was assumed that function $f$ satisfies a one-sided Lipschitz condition with respect to the last two variables with corresponding functions instead of constants. Note that the special case when $f$ is monotone nonincreasing (with respect to the last two variables) is not discussed in paper [4] and is of particular interest. Moreover, at the end of this paper we formulate conditions under which problem (1.1) has a unique solution. This paper extends some results of [4]. 
The plan of this paper is as follows. In Section 3, we discuss problem (1.1) for the case when $r \leq 0$. Theorem 3.1 says about extremal quasisolutions of problem (1.1). Example 3.2 illustrates that assumptions of Theorem 3.1 are satisfied, so the problem (3.13), from this example, has extremal quasisolutions which are the limit of sequences $\left\{y_{n}, z_{n}\right\}$. To obtain an approximate extremal quasisolutions we can use the elements of sequences $\left\{y_{n}, z_{n}\right\}$. Using numerical methods we can find numerical approximations $\tilde{y}_{n}, \tilde{z}_{n}$ of $y_{n}, z_{n}$, respectively. The graphs of some $\tilde{y}_{n}, \tilde{z}_{n}$ are given in Figure 3.1. In Section 4, we investigate problems having more deviating arguments. Also an example and graphs of numerical approximations of $\tilde{y}_{n}, \tilde{z}_{n}$ are given. In Section 6, we combined results of this paper with corresponding results of [4]. Example 6.5 shows the results obtained. In the last section, we investigate the problem when the minimal and maximal quasisolutions are equal, so when our problem has a unique solution.

\section{Lemmas and definitions}

We need some lemmas which are useful in this paper.

It is easy to show the following.

Lemma 2.1. Let $p \in C^{2}(J, \mathbb{R})$ and

$$
\begin{gathered}
p^{\prime \prime}(t) \geq 0 \quad \text { for } t \in J, \\
p(0) \leq 0, \quad p(T) \leq 0 .
\end{gathered}
$$

Then $p(t) \leq 0$ on $J$.

It is a well-known fact which follows from Green function properties that the following holds.

LEMMA 2.2. Let

$$
G(t, s)=-\frac{1}{T} \begin{cases}(T-t) s & \text { for } 0 \leq s \leq t \leq T \\ (T-s) t & \text { for } 0 \leq t \leq s \leq T\end{cases}
$$

Let $h: J \rightarrow \mathbb{R}$ be integrable on $J$. Then the problem

$$
u^{\prime \prime}(t)=h(t), \quad u(0)=0, \quad u(T)=\beta
$$

has exactly one solution given by

$$
u(t)=\int_{0}^{T} G(t, s) h(s) d s+\frac{\beta}{T} t .
$$

We assume in all definitions below that $r \leq 0$. 
Definition 2.3. A pair of functions $y_{0}, z_{0} \in C^{2}(J, \mathbb{R})$ is called coupled lower and upper solutions of (1.1) if

$$
\begin{aligned}
& y_{0}^{\prime \prime}(t) \geq F z_{0}(t), \quad y_{0}(0) \leq 0, \quad y_{0}(T) \leq r z_{0}(\gamma), \\
& z_{0}^{\prime \prime}(t) \leq F y_{0}(t), \quad z_{0}(0) \geq 0, \quad z_{0}(T) \geq r y_{0}(\gamma),
\end{aligned}
$$

where $t \in J$.

Definition 2.4. A pair of functions $Y, Z \in C^{2}(J, \mathbb{R})$ is called coupled quasisolutions of (1.1) if

$$
\begin{array}{lll}
Y^{\prime \prime}(t)=F Z(t), & Y(0)=0, & Y(T)=r Z(\gamma), \\
Z^{\prime \prime}(t)=F Y(t), & Z(0)=0, & Z(T)=r Y(\gamma),
\end{array}
$$

where $t \in J$.

Let $f, g \in C^{2}(J, \mathbb{R})$ and $f(t) \leq g(t)$ for $t \in J$. We will say that a function $e \in C^{2}(J, \mathbb{R})$ belongs to segment $[f, g]$ if $f(t) \leq e(t) \leq g(t)$ for $t \in J$.

Definition 2.5. Let a pair $(U, V)$ be a coupled quasisolutions of $(1.1)$. $(U, V)$ are called minimal and maximal coupled quasisolutions of (1.1) if for any else $\bar{U}, \bar{V}$ coupled quasisolutions of (1.1), it holds that $U(t) \leq \bar{U}(t), \bar{V}(t) \leq V(t), t \in J$.

Let $u, v \in C^{2}(J, \mathbb{R})$ satisfy $u(t) \leq v(t)$ for $t \in J$. Coupled quasisolutions $U, V$ of $(1.1)$ are called minimal and maximal coupled quasisolutions in segment $[u, v]$ if $u(t) \leq U(t)$, $V(t) \leq v(t)$ for $t \in J$ and for any else $(Y, Z)$ coupled quasisolutions of $(1.1)$, such as $u(t) \leq$ $Y(t), Z(t) \leq v(t)$ for $t \in J$, it holds that $U(t) \leq Y(t)$ and $Z(t) \leq V(t), t \in J$.

Remark 2.6. Note that if a function $y$ is a solution of $(1.1)$, then the pair $(y, y)$ will be coupled quasisolutions of (1.1).

\section{Main results if $r \leq 0$}

Now we formulate conditions which guarantee that problem (1.1) has extremal quasisolutions.

Theorem 3.1. Let $r \leq 0, f \in C(J \times \mathbb{R} \times \mathbb{R}, \mathbb{R})$, and $\alpha \in C(J, J)$. Let $y_{0}$, $z_{0}$ be coupled lower and upper solutions of (1.1) and $y_{0}(t) \leq z_{0}(t), t \in J$. Moreover, assume that

$$
f\left(t, \bar{u}_{1}, \bar{v}_{1}\right)-f\left(t, u_{1}, v_{1}\right) \leq 0
$$

for $y_{0}(t) \leq \bar{u}_{1} \leq u_{1} \leq z_{0}(t), y_{0}(\alpha(t)) \leq \bar{v}_{1} \leq v_{1} \leq v_{0}(\alpha(t))$.

Then problem (1.1) has in segment $\left[y_{0}, z_{0}\right]$ the minimal and maximal coupled quasisolutions. 
4 Second-order differential equations

Proof. Let

$$
\begin{gathered}
y_{n}^{\prime \prime}(t)=F z_{n-1}(t), \quad t \in J, \\
y_{n}(0)=0, \quad y_{n}(T)=r z_{n-1}(\gamma), \\
z_{n}^{\prime \prime}(t)=F y_{n-1}(t), \quad t \in J, \\
z_{n}(0)=0, \quad z_{n}(T)=r y_{n-1}(\gamma)
\end{gathered}
$$

for $n \in \mathbb{N}=\{1,2,3, \ldots\}$. If $n=1$, then from Lemma 2.2 we know that problems (3.2) have unique solutions $y_{1}$ and $z_{1}$.

We need to show that

$$
y_{0}(t) \leq y_{1}(t) \leq z_{1}(t) \leq z_{0}(t), \quad t \in J
$$

Let $p(t)=y_{0}(t)-y_{1}(t)$. From the definition of coupled lower and upper solutions, we get $p(0) \leq 0-0=0, p(T) \leq r z_{0}(\gamma)-r z_{0}(\gamma)=0$, and

$$
p^{\prime \prime}(t)=y_{0}^{\prime \prime}(t)-y_{1}^{\prime \prime}(t) \geq F z_{0}(t)-F z_{0}(t)=0 \text {. }
$$

This and Lemma 2.1 give us $p(t) \leq 0$ for $t \in[0, T]$. From this we obtain $y_{0}(t) \leq y_{1}(t)$ for $t \in J$. By the same way we can show that $z_{1}(t) \leq z_{0}(t)$ for $t \in J$.

Now we will show that $y_{1}(t) \leq z_{1}(t)$ for $t \in J$. Let $p(t)=y_{1}(t)-z_{1}(t)$. Then we have $p(0)=0, p(T)=r\left[z_{0}(t)-y_{0}(t)\right] \leq 0$, and from (3.1), we get

$$
p^{\prime \prime}(t)=F z_{0}(t)-F y_{0}(t) \geq 0
$$

In view of Lemma 2.1, we obtain $y_{1}(t) \leq z_{1}(t)$ for $t \in J$. It shows that (3.3) holds.

There is no problem to show that $y_{1}$ and $z_{1}$ are coupled lower and upper solutions of (1.1).

By induction in $n$, we obtain the relation

$$
y_{0}(t) \leq \cdots \leq y_{n-1}(t) \leq y_{n}(t) \leq z_{n}(t) \leq z_{n-1}(t) \leq \cdots \leq z_{0}(t)
$$

for $t \in J, n \in \mathbb{N}$.

There is no problem to show that sequences $\left\{y_{n}\right\},\left\{z_{n}\right\}$ are equicontinuous and bounded on $J$. The Arzeli-Ascoli theorem guarantees the existence of subsequences $\left\{y_{n_{k}}\right\},\left\{z_{n_{k}}\right\}$ and functions $y, z \in C(J, \mathbb{R})$ with $\left\{y_{n_{k}}\right\},\left\{z_{n_{k}}\right\}$ converging uniformly on $J$ to $y, z$, respectively, when $n_{k} \rightarrow \infty$. However, since the sequences $\left\{y_{n}\right\},\left\{z_{n}\right\}$ are monotonic, we conclude that whole sequences $\left\{y_{n}\right\},\left\{z_{n}\right\}$ converge uniformly on $J$ to $y, z$, respectively. If 
$n \rightarrow \infty$ in integral equations for $y_{n}$ and $z_{n}$, we get

$$
\begin{array}{lll}
y(t)=\int_{0}^{T} G(t, s) F z(s) d s+\frac{t}{T} r z(\gamma), & y(0)=0, & y(T)=r z(\gamma), \\
z(t)=\int_{0}^{T} G(t, s) F y(s) d s+\frac{t}{T} r y(\gamma), & z(0)=0, & z(T)=r y(\gamma) .
\end{array}
$$

From above it is easy to show that

$$
\begin{gathered}
y^{\prime \prime}(t)=F z(t), \quad y(0)=0, \quad y(T)=r z(\gamma), \\
z^{\prime \prime}(t)=F y(t), \quad z(0)=0, \quad z(T)=r y(\gamma), \quad t \in J .
\end{gathered}
$$

It means that $y, z$ are coupled quasisolutions of problem (1.1). Now we have to prove that $(y, z)$ are minimal and maximal coupled quasisolutions of problem (1.1) in segment $\left[y_{0}, z_{0}\right]$. Let $(\bar{y}, \bar{z})$ be coupled quasisolutions of (1.1) such that

$$
y_{m}(t) \leq \bar{y}(t), \quad \bar{z}(t) \leq z_{m}(t), \quad t \in J
$$

for some $m \in \mathbb{N}$. Put $p(t)=y_{m+1}(t)-\bar{y}(t), t \in J$. Hence, $p(0)=0$,

$$
\begin{gathered}
p(T)=r z_{m}(\gamma)-r \bar{z}(\gamma)=r\left[z_{m}(\gamma)-\bar{z}(\gamma)\right] \leq 0 \\
p^{\prime \prime}(t)=F z_{m}(t)-F \bar{z}(t) \geq 0
\end{gathered}
$$

By Lemma 2.1, we get $p(t) \leq 0$ so $y_{m+1}(t) \leq \bar{y}(t)$ for $t \in J$. By a similar way we can show that $\bar{z}(t) \leq z_{m+1}(t), t \in J$. By induction, we obtain

$$
y_{n}(t) \leq \bar{y}(t), \quad \bar{z}(t) \leq z_{n}(t), \quad \text { for } n \in \mathbb{N} .
$$

If $n \rightarrow \infty$, it yields

$$
y(t) \leq \bar{y}(t), \quad \bar{z}(t) \leq z(t), \quad t \in J .
$$

It shows that $(y, z)$ are minimal and maximal coupled quasisolutions of problem (1.1) in segment $\left[y_{0}, z_{0}\right]$.

Example 3.2. Let us consider a problem

$$
\begin{gathered}
x^{\prime \prime}(t)=\sin (x(t))+x(0.9 t)+\frac{1}{32}, \quad t \in J=[0,1], \\
x(0)=0, \quad x(1)=-x(0.5) .
\end{gathered}
$$




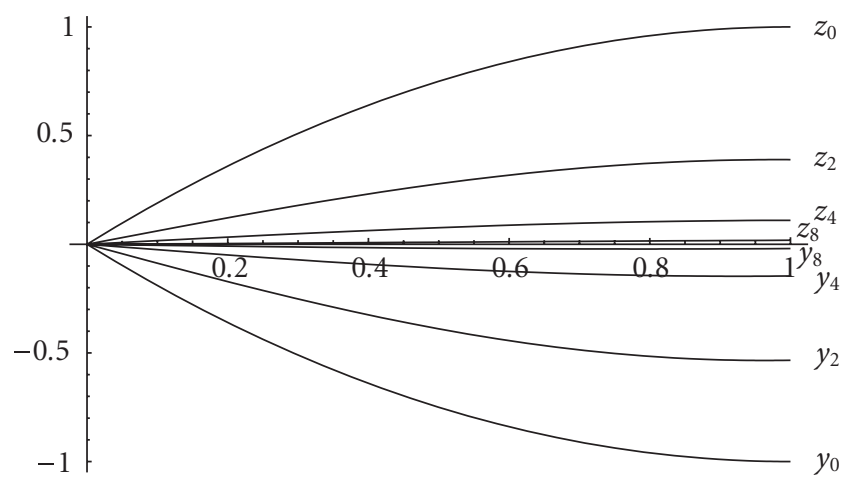

Figure 3.1. Some iterations for Example 3.2.

Put $y_{0}(t)=t(t-2), z_{0}(t)=-t(t-2)$. Then

$$
\begin{aligned}
y_{0}(0)= & z_{0}(0)=0, \quad y_{0}(1)=-1<-\frac{3}{4}=-z_{0}(0.5), \quad z_{0}(1)=1>\frac{3}{4}=-y_{0}(0.5), \\
& \sin (-t(t-2))-0.9 t(0.9 t-2)+\frac{1}{32} \leq \sin (1)+1+\frac{1}{32}<2=y_{0}^{\prime \prime}(t), \\
& \sin (t(t-2))+0.9 t(0.9 t-2)+\frac{1}{32} \geq \sin (-1)-1+\frac{1}{32}>-2=z_{0}^{\prime \prime}(t) .
\end{aligned}
$$

We show that $y_{0}, z_{0}$ are coupled lower and upper solutions of (3.13). Indeed, condition (3.1) holds. In view of Theorem 3.1, problem (3.13) has, in segment $\left[y_{0}, z_{0}\right]$, the minimal and maximal coupled quasisolutions.

In Figure 3.1, we see numerical results of some iterations algorithm from Theorem 3.1. Numerical solutions have been found by Mathematica 4.0 . Solutions are interpolated by Lagrange interpolating polynomials to obtain values for deviating arguments. In this picture we have only iterations $y_{i}, z_{i}$ for $i=0,2,4,8$.

\section{Generalization}

Let us consider a boundary value problem

$$
\begin{gathered}
x^{\prime \prime}(t)=g\left(t, x(t), x\left(\alpha_{1}(t)\right), \ldots, x\left(\alpha_{p}(t)\right)\right) \equiv F x(t), \quad t \in J=[0, T], \\
x(0)=0, \quad x(T)=r x(\gamma) \quad \text { for } \gamma \in(0, T),
\end{gathered}
$$

where $g \in C\left(J \times \mathbb{R}^{p+1}, \mathbb{R}\right), r, \gamma$ are fixed numbers and functions $\alpha_{i} \in C(J, J)$ for $i=1, \ldots, p$. Definitions of coupled lower and upper solutions, coupled quasisolutions, and minimal 
and maximal coupled quasisolutions of problem (4.1) are analogy of Definitions 2.3, 2.4, and 2.5. Now we write analogue of Theorem 3.1 for the problem (4.1). We omit the proof of this theorem because it is similar to the one of Theorem 3.1.

Theorem 4.1. Let $r \leq 0, g \in C\left(J \times \mathbb{R}^{p+1}, \mathbb{R}\right)$, and $\alpha_{i} \in C(J, J)$ for $i=1, \ldots, p$. Let $y_{0}, z_{0}$ be coupled lower and upper solutions of (4.1) and $y_{0}(t) \leq z_{0}(t), t \in J$. Moreover, assume that

$$
g\left(t, \bar{u}_{1}, \bar{v}_{1}, \ldots, \bar{v}_{p}\right)-g\left(t, u_{1}, v_{1}, \ldots, v_{p}\right) \leq 0
$$

for $y_{0}(t) \leq \bar{u}_{1} \leq u_{1} \leq z_{0}(t), y_{0}\left(\alpha_{i}(t)\right) \leq \bar{v}_{i} \leq v_{i} \leq v_{0}\left(\alpha_{i}(t)\right)$ for $i=1, \ldots, p$.

Then problem (4.1) has, in segment $\left[y_{0}, z_{0}\right]$, the minimal and maximal coupled quasisolutions.

Example 4.2. For $J=[0,1]$, let us consider a problem

$$
\begin{gathered}
x^{\prime \prime}(t)=0.4 \sin (x(t))+0.2 x(0.9 t)+0.5 \exp (x(\sqrt{t}))+\frac{1}{32}, \quad t \in J, \\
x(0)=0, \quad x(1)=-x(0.5) .
\end{gathered}
$$

Note that $\alpha_{1}(t)=0.9 t, \alpha_{2}(t)=\sqrt{t}$. Put $y_{0}(t)=t(t-2), z_{0}(t)=-t(t-2)$. Then $y_{0}(0)=$ $z_{0}(0)=0, y_{0}(1)<-z_{0}(0.5), z_{0}(1)>-y_{0}(0.5)$, and

$$
\begin{aligned}
& 0.4 \sin (-t(t-2))-0.2(0.9 t(0.9 t-2))+0.5 \exp (-\sqrt{t}(\sqrt{t}-2))+\frac{1}{32} \\
& \leq 0.4 \sin (1)+0.2+0.5 \exp (1)+\frac{1}{32} \approx 1.93<2=y_{0}^{\prime \prime}(t) \\
& 0.4 \sin (t(t-2))+0.18 t(0.9 t-2)+0.5 \exp (\sqrt{t}(\sqrt{t}-2))+\frac{1}{32} \\
& \geq 0.4 \sin (-1)-0.2+0.5 \exp (-1)+\frac{1}{32} \approx-0.32>-2=z_{0}^{\prime \prime}(t) .
\end{aligned}
$$

We see that $y_{0}, z_{0}$ are coupled lower and upper solutions of (4.3). Indeed, $g$ satisfies condition (4.2). In view of Theorem 4.1, problem (4.3) has in segment $\left[y_{0}, z_{0}\right]$ the minimal and maximal coupled quasisolutions. On Figure 4.1 we see results of first three iterations.

\section{Result for $r>0$}

We would like to transfer proof techniques used before to problem (1.1) with $r>0$. To get a similar result we have to change definitions.

Definition 5.1. A pair of functions $y_{0}, z_{0} \in C^{2}(J, \mathbb{R})$ are called coupled lower and upper solutions of (1.1) if

$$
\begin{aligned}
& y_{0}^{\prime \prime}(t) \geq F z_{0}(t), \quad y_{0}(0) \leq 0, \quad y_{0}(T) \leq r y_{0}(\gamma), \\
& z_{0}^{\prime \prime}(t) \leq F y_{0}(t), \quad z_{0}(0) \geq 0, \quad z_{0}(T) \geq r z_{0}(\gamma),
\end{aligned}
$$

where $t \in J$. 
8 Second-order differential equations

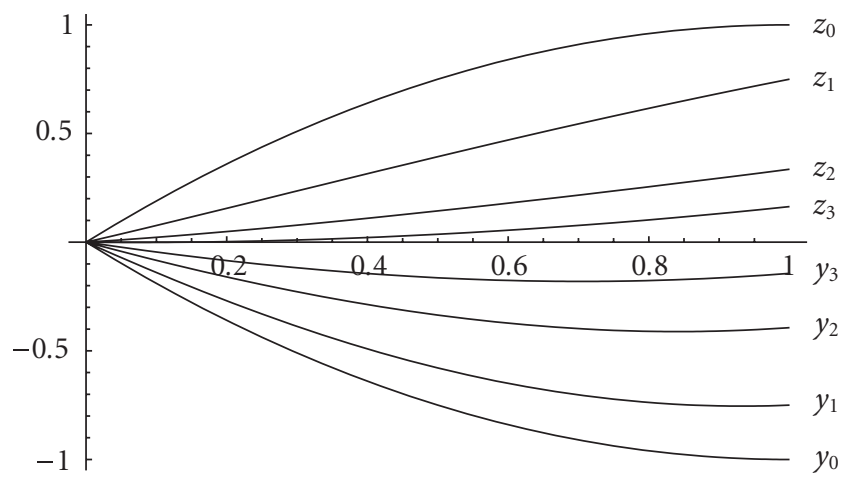

Figure 4.1. Result of three iterations in Example 4.2.

Definition 5.2. A pair of functions $Y, Z \in C^{2}(J, \mathbb{R})$ are called coupled quasisolutions of (1.1) if

$$
\begin{array}{lll}
Y^{\prime \prime}(t)=F Z(t), & Y(0)=0, & Y(T)=r Y(\gamma), \\
Z^{\prime \prime}(t)=F Y(t), & Z(0)=0, & Z(T)=r Z(\gamma),
\end{array}
$$

where $t \in J$.

We can prove Theorem 5.3 by the same way as we proved Theorem 3.1.

Theorem 5.3. Let $f \in C(J \times \mathbb{R} \times \mathbb{R}, \mathbb{R}), r>0$, and $\alpha \in C(J, J)$. Let $y_{0}$, $z_{0}$ be coupled lower and upper solutions of (1.1) and $y_{0}(t) \leq z_{0}(t), t \in J$. Moreover, assume that

$$
f\left(t, \bar{u}_{1}, \bar{v}_{1}\right)-f\left(t, u_{1}, v_{1}\right) \leq 0
$$

for $y_{0}(t) \leq \bar{u}_{1} \leq u_{1} \leq z_{0}(t), y_{0}(\alpha(t)) \leq \bar{v}_{1} \leq v_{1} \leq v_{0}(\alpha(t))$.

Then problem (1.1) has, in segment $\left[y_{0}, z_{0}\right]$, the minimal and maximal coupled quasisolutions.

\section{Combination of coupled quasisolutions}

It is turned out that we can combine some results of [4] with this work. In [4], it is assumed that $f$ satisfies one-side Lipschitz condition with corresponding functional coefficients.

Theorem 6.1 (see [4, Theorem 5]). Let $f \in C(J \times \mathbb{R} \times \mathbb{R}, \mathbb{R}), r \leq 0$, and $\alpha \in C(J, J)$. Let $y_{0}, z_{0}$ be coupled lower and upper solutions of (1.1) and $y_{0}(t) \leq z_{0}(t), t \in J$. Moreover, 
assume that

$$
\begin{gathered}
M, N \in C(J,[0, \infty)), \quad M(t)>0, t \in(0, T), \\
\rho \equiv \max \left\{\int_{0}^{T}\left(\int_{s}^{T}[M(t)+N(t)] d t\right) d s, \int_{0}^{T}\left(\int_{0}^{s}[M(t)+N(t)] d t\right) d s\right\} \leq 1, \\
f\left(t, \bar{e}_{1}, \bar{r}_{1}\right)-f\left(t, e_{1}, r_{1}\right) \geq-M(t)\left[e_{1}-\bar{e}_{1}\right]-N(t)\left[r_{1}-\bar{r}_{1}\right],
\end{gathered}
$$

where $y_{0}(t) \leq \bar{e}_{1} \leq e_{1} \leq z_{0}(t), y_{0}(\alpha(t)) \leq \bar{r}_{1} \leq r_{1} \leq z_{0}(\alpha(t))$.

Then problem (1.1) has, in segment $\left[y_{0}, z_{0}\right]$, the minimal and maximal coupled quasisolutions.

Let us introduce the following operator:

$$
F(x, y)(t)=f(t, x(t), x(\alpha(t)), y(t), y(\beta(t))) \text {, }
$$

where $\alpha, \beta \in C(J, J)$.

Now we consider a problem

$$
\begin{gathered}
x^{\prime \prime}(t)=F(x, x)(t), \quad t \in J=[0, T], \\
x(0)=0, \quad x(T)=r x(\gamma) \quad \text { with } \gamma \in(0, T), r \leq 0,
\end{gathered}
$$

where $f \in C\left(J \times \mathbb{R}^{4}, \mathbb{R}\right), r, \gamma$ are fixed numbers and $\alpha, \beta \in C(J, J)$.

We will combine definitions of coupled lower and upper solutions with coupled lower and upper solutions.

Definition 6.2. A pair of functions $y_{0}, z_{0} \in C^{2}(J, \mathbb{R})$ is called coupled lower and upper solutions of (6.5) if

$$
\begin{aligned}
& y_{0}^{\prime \prime}(t) \geq F\left(z_{0}, y_{0}\right)(t), \quad y_{0}(0) \leq 0, \quad y_{0}(T) \leq r z_{0}(\gamma), \\
& z_{0}^{\prime \prime}(t) \leq F\left(y_{0}, z_{0}\right)(t), \quad z_{0}(0) \geq 0, \quad z_{0}(T) \geq r y_{0}(\gamma),
\end{aligned}
$$

where $t \in J$.

Definition 6.3. A pair of functions $Y, Z \in C^{2}(J, \mathbb{R})$ is called coupled quasisolutions of $(6.5)$ if

$$
\begin{aligned}
& Y^{\prime \prime}(t)=F(Z, Y)(t), \quad Y(0)=0, \quad Y(T)=r Z(\gamma), \\
& Z^{\prime \prime}(t)=F(Y, Z)(t), \quad Z(0)=0, \quad Z(T)=r Y(\gamma),
\end{aligned}
$$

where $t \in J$ and $0<\gamma<T$.

Theorem 6.4. Let $f \in C\left(J \times \mathbb{R}^{4}, \mathbb{R}\right), r \leq 0$, and $\alpha, \beta \in C(J, J)$. Let $y_{0}$, $z_{0}$ be coupled lower and upper solutions of (6.5) and $y_{0}(t) \leq z_{0}(t), t \in J$. Moreover, assume that

$$
f\left(t, \bar{u}_{1}, \bar{v}_{1}, u_{2}, u_{3}\right)-f\left(t, u_{1}, v_{1}, u_{2}, u_{3}\right) \leq 0,
$$


where $y_{0}(t) \leq \bar{u}_{1} \leq u_{1} \leq z_{0}(t), y_{0}(\alpha(t)) \leq \bar{v}_{1} \leq v_{1} \leq z_{0}(\alpha(t))$, and

$$
f\left(t, w_{1}, w_{2}, \bar{e}_{1}, \bar{r}_{1}\right)-f\left(t, w_{1}, w_{2}, e_{1}, r_{1}\right) \geq-M(t)\left[e_{1}-\bar{e}_{1}\right]-N(t)\left[r_{1}-\bar{r}_{1}\right]
$$

where $y_{0}(t) \leq \bar{e}_{1} \leq e_{1} \leq z_{0}(t), y_{0}(\beta(t)) \leq \bar{r}_{1} \leq r_{1} \leq z_{0}(\beta(t))$ and for $M, N$ conditions (6.1) and (6.2) hold.

Then problem (6.5) has, in segment $\left[y_{0}, z_{0}\right]$, the minimal and maximal coupled quasisolutions.

To prove this theorem we apply the way of this paper combined with [4] and therefore we omit the proof. Note that $y_{n}$ and $z_{n}$ are defined by

$$
\begin{gathered}
y_{n}^{\prime \prime}(t)=F\left(z_{n-1}, y_{n-1}\right)(t)+M(t)\left[y_{n}(t)-y_{n-1}(t)\right]+N(t)\left[y_{n}(\beta(t))-y_{n-1}(\beta(t))\right], \quad t \in J, \\
y_{n}(0)=0, \quad y_{n}(T)=r z_{n-1}(\gamma), \\
z_{n}^{\prime \prime}(t)=F\left(y_{n-1}, z_{n-1}\right)(t)+M(t)\left[z_{n}(t)-z_{n-1}(t)\right]+N(t)\left[z_{n}(\beta(t))-z_{n-1}(\beta(t))\right], \quad t \in J, \\
z_{n}(0)=0, \quad z_{n}(T)=r y_{n-1}(\gamma) .
\end{gathered}
$$

Example 6.5. Let us consider a problem which is combination of examples from this paper and from [4], so we omit checking the assumptions about $f$,

$$
\begin{gathered}
x^{\prime \prime}(t)=\sin (x(t))+x(0.9 t)+\frac{1}{32}+x(t) \sin (t)+0.4 x(0.5 t) \cos (t)-t \sin (t), \quad t \in J=[0,1] \\
x(0)=0, \quad x(1)=-x(0.5) .
\end{gathered}
$$

Put $y_{0}(t)=t(t-2), z_{0}(t)=-t(t-2)$. It is easy (just like before) to show that $y_{0}, z_{0}$ are coupled lower and upper solutions of (6.11). Thus problem (6.11) has, in segment $\left[y_{0}, z_{0}\right]$, the minimal and maximal coupled quasisolutions. On Figure 6.1 we see some chosen pairs of numerical approximations of quasisolutions of problem (6.11).

Remark 6.6. There is no problem to investigate problem (6.5) when $r>0$.

\section{From minimal and maximal quasisolutions to solution}

We can ask: what conditions will we assume to obtain $y=z$ ? In all previous theorems we got that minimal and maximal quasisolutions $y$ and $z$ satisfying $y(t) \leq z(t), t \in J$. Now we put $p(t)=z(t)-y(t)$ and try to find conditions which guarantee that $p(t)=0$. Those conditions should not be contradictory with the previous assumptions. First of all, we prove a lemma which will be useful to show that $p(t)=0$. 


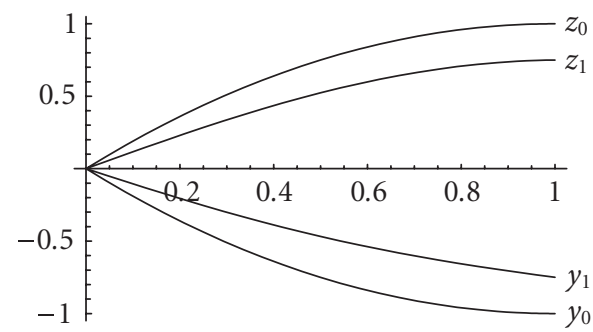

(a)

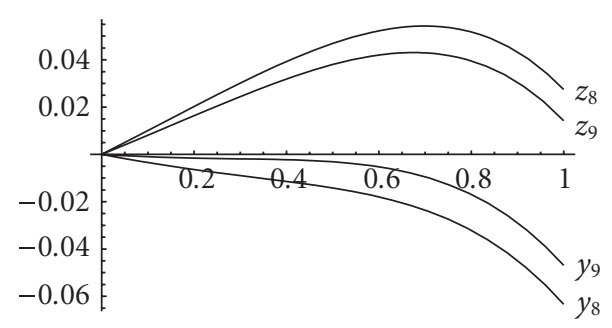

(c)

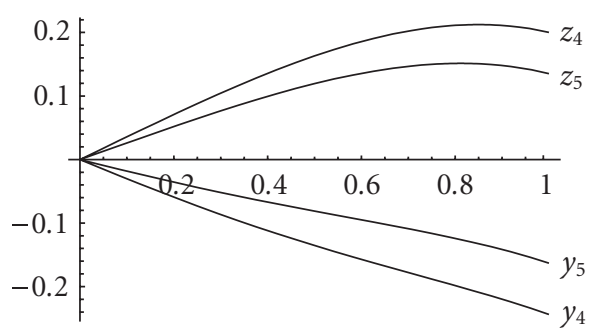

(b)

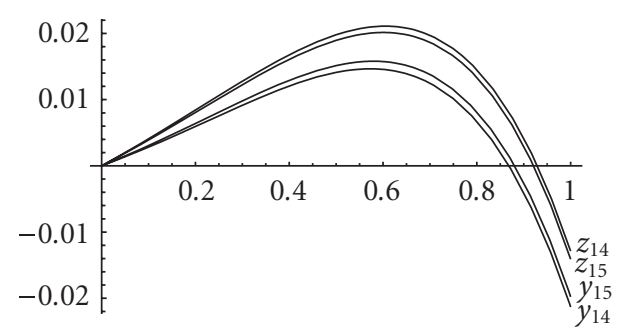

(d)

Figure 6.1. Chosen pairs of quasisolutions of problem (6.11).

Lemma 7.1. Assume that $\gamma \in(0, T)$ and $0 \leq k \gamma<T$. Let $p \in C^{2}(J, \mathbb{R}), B \in C\left(C^{2}(J, \mathbb{R}) \times\right.$ $J, \mathbb{R})$, and

$$
\begin{gathered}
p^{\prime \prime}(t) \geq B(p, t), \quad t \in J, \\
p(0) \leq 0, \quad p(T) \leq k p(\gamma) .
\end{gathered}
$$

Then function $p$ satisfies the following inequality:

$$
p(t) \leq \frac{k t}{T-k \gamma} \int_{0}^{\gamma} \int_{0}^{s} B(p, \tau) d \tau d s+\int_{0}^{t} \int_{0}^{s} B(p, \tau) d \tau d s-\frac{t}{T-k \gamma} \int_{0}^{T} \int_{0}^{s} B(p, \tau) d \tau d s .
$$

Proof. We replace problem (7.1) by

$$
\begin{gathered}
p^{\prime \prime}(t)=B(p, t)+A, \quad t \in J, \\
p(0)=a, \quad p(T)=k p(\gamma)+b
\end{gathered}
$$

with $A \geq 0, a \leq 0, b \leq 0$. Integrating it two times on $[0, t]$, we obtain

$$
p(t)=a+p^{\prime}(0) t+\frac{1}{2} A t^{2}+\int_{0}^{t} \int_{0}^{s} B(p, \tau) d \tau d s, \quad t \in J .
$$


To calculate $p^{\prime}(0)$, we have to use boundary conditions. Thus

$$
\begin{aligned}
p(t)= & \frac{1}{2} A t\left(\frac{\gamma^{2} k-T^{2}}{T-k \gamma}+t\right)+\frac{k t}{T-k \gamma} \int_{0}^{\gamma} \int_{0}^{s} B(p, \tau) d \tau d s \\
& +\int_{0}^{t} \int_{0}^{s} B(p, \tau) d \tau d s-\frac{t}{T-k \gamma} \int_{0}^{T} \int_{0}^{s} B(p, \tau) d \tau d s \\
& +a\left[1+\frac{t(k-1)}{T-k \gamma}\right]+\frac{b t}{T-k \gamma} .
\end{aligned}
$$

Note that if $k \geq 1$, then $1+t(k-1) /(T-k \gamma) \geq 1$. Now if $0 \leq k<1$, then

$$
1+\frac{t(k-1)}{T-k \gamma} \geq 1+\frac{T(k-1)}{T-k \gamma}=\frac{k(T-\gamma)}{T-k \gamma} \geq 0 .
$$

In view of the inequality $\gamma^{2} k \leq \gamma T k$, assumptions $a \leq 0, b \leq 0$, and (7.5), we obtain

$$
\begin{aligned}
p(t) \leq & \frac{1}{2} A t(t-T)+\frac{k t}{T-k \gamma} \int_{0}^{\gamma} \int_{0}^{s} B(p, \tau) d \tau d s \\
& +\int_{0}^{t} \int_{0}^{s} B(p, \tau) d \tau d s-\frac{t}{T-k \gamma} \int_{0}^{T} \int_{0}^{s} B(p, \tau) d \tau d s .
\end{aligned}
$$

Hence we have (7.2) since $A \geq 0$. It ends the proof.

To use this lemma we will additionally assume that $f$ satisfies one-side Lipschitz condition with corresponding functional coefficients.

Theorem 7.2. Assume that all assumptions of Theorem 6.4 hold. Let $T>-r \gamma$. In addition, assume that there exist functions $L_{1}, L_{2}, L_{3}, L_{4} \in C\left(J, \mathbb{R}^{+}\right)$such that

$$
\begin{gathered}
\frac{T}{T-k \gamma} \int_{0}^{T}\left(\int_{0}^{s}\left[L_{1}(t)+L_{2}(t)+L_{3}(t)+L_{4}(t)\right] d t\right) d s<1, \\
-L_{1}(t)\left[u_{1}-\bar{u}_{1}\right]-L_{2}(t)\left[v_{1}-\bar{v}_{1}\right] \leq f\left(t, \bar{u}_{1}, \bar{v}_{1}, u_{2}, u_{3}\right)-f\left(t, u_{1}, v_{1}, u_{2}, u_{3}\right),
\end{gathered}
$$

where $y_{0}(t) \leq \bar{u}_{1} \leq u_{1} \leq z_{0}(t), y_{0}(\alpha(t)) \leq \bar{v}_{1} \leq v_{1} \leq z_{0}(\alpha(t))$, and

$$
L_{3}(t)\left[e_{1}-\bar{e}_{1}\right]+L_{4}(t)\left[r_{1}-\bar{r}_{1}\right] \geq f\left(t, w_{1}, w_{2}, \bar{e}_{1}, \bar{r}_{1}\right)-f\left(t, w_{1}, w_{2}, e_{1}, r_{1}\right),
$$

where $y_{0}(t) \leq \bar{e}_{1} \leq e_{1} \leq z_{0}(t), y_{0}(\beta(t)) \leq \bar{r}_{1} \leq r_{1} \leq z_{0}(\beta(t))$.

Then problem (6.5) has, in segment $\left[y_{0}, z_{0}\right]$, exactly one solution.

Proof. From Theorem 6.4, we know that problem (6.5) has, in segment $\left[y_{0}, z_{0}\right]$, the minimal and maximal coupled quasisolutions $y$ and $z$ and $y(t) \leq z(t)$ on $J$. Let $p(t)=z(t)-$ $y(t)$, so $p(t) \geq 0, t \in J$. By definitions of minimal and maximal coupled quasisolutions, we obtain $p(0)=0, p(\gamma)=-r p(\gamma)$, and $p^{\prime \prime}(t)=F(y, z)(t)-F(z, y)(t)$. In view of (7.9) 
and (7.10), we get

$$
\begin{aligned}
p^{\prime \prime}(t) & =F(y, z)(t)-F(z, z)(t)+F(z, z)(t)-F(z, y)(t) \\
& \geq-L_{1}(t) p(t)-L_{2}(t) p(\alpha(t))-L_{3}(t) p(t)-L_{4}(t) p(\beta(t)) \equiv B(p, t) .
\end{aligned}
$$

It is obvious that $B(p, t) \leq 0$ for $t \in J$. From Lemma 7.1, we obtain

$$
p(t) \leq \frac{k t}{T-k \gamma} \int_{0}^{\gamma} \int_{0}^{s} B(p, \tau) d \tau d s+\int_{0}^{t} \int_{0}^{s} B(p, \tau) d \tau d s-\frac{t}{T-k \gamma} \int_{0}^{T} \int_{0}^{s} B(p, \tau) d \tau d s
$$

with $k=-r$.

Note that the first two elements of (7.12) are negative, so we omit them. Hence

$$
p(t) \leq-\frac{t}{T-k \gamma} \int_{0}^{T} \int_{0}^{s} B(p, \tau) d \tau d s, \quad t \in J .
$$

Suppose, that $\max _{t \in J} p(t)=p\left(t_{1}\right)=D>0$. From (7.13), we obtain

$$
\begin{aligned}
D & \leq \frac{t_{1}}{T-k \gamma} \int_{0}^{T} \int_{0}^{s}\left[\left(L_{1}(t)+L_{3}(t)\right) p\left(t_{1}\right)+L_{2}(t) p\left(\alpha\left(t_{1}\right)\right)+L_{4}(t) p\left(\beta\left(t_{1}\right)\right)\right] d t d s \\
& \leq \frac{T D}{T-k \gamma} \int_{0}^{T} \int_{0}^{s}\left[L_{1}(t)+L_{2}(t)+L_{3}(t)+L_{4}(t)\right] d t d s,
\end{aligned}
$$

so

$$
D\left[1-\frac{T}{T-k \gamma} \int_{0}^{T}\left(\int_{0}^{s}\left[L_{1}(t)+L_{2}(t)+L_{3}(t)+L_{4}(t)\right] d t\right) d s\right] \leq 0 .
$$

By condition (7.8), we get $D \leq 0$. It is a contradiction.

It proves that $p(t)=0$ for $t \in J$. Thus $y \equiv z$ and $y$ is a solution of problem (6.5).

Remark 7.3. For example, if $L_{i}(t)=L_{i}, i=1,2,3,4$, then condition (7.8) takes the form

$$
L_{1}+L_{2}+L_{3}+L_{4}<2 \frac{T+r \gamma}{T^{3}}
$$

Example 7.4. Let us consider a problem like in Example 6.5 with a little modification of the boundary condition, namely,

$$
\begin{gathered}
x^{\prime \prime}(t)=\sin (x(t))+x(0.9 t)+\frac{1}{32}+x(t) \sin (t)+0.4 x(0.5 t) \cos (t)-t \sin (t), \quad t \in J=[0,1] \\
x(0)=0, \quad x(1)=-x(0.3) .
\end{gathered}
$$

Put $y_{0}(t)=t(t-2), z_{0}(t)=-t(t-2)$. All assumptions of Theorem 6.4 are satisfied. Now we have to show that additional assumptions from Theorem 7.2 hold. 


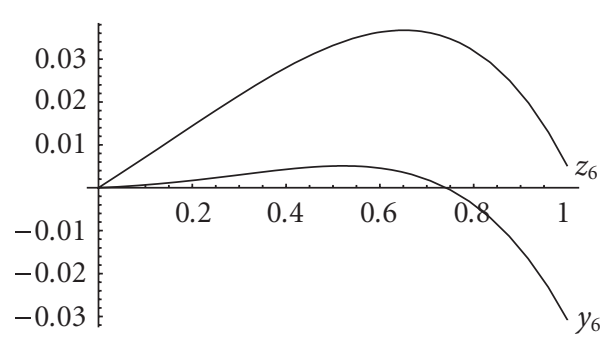

(a)

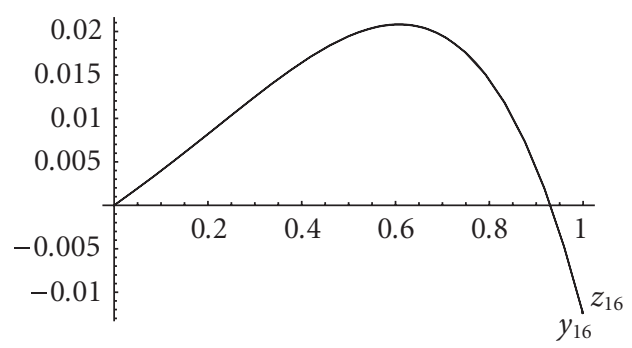

(b)

Figure 7.1. Iterations number 6 and 16.

Take

$$
\begin{gathered}
f_{1}(t, u, v)=\sin (u)+v+\frac{1}{32} \\
f_{2}(t, e, r)=e \sin (t)+0.4 r \cos (t)-t \sin (t) .
\end{gathered}
$$

Let $\bar{u}_{1} \leq u_{1}$ and $\bar{v}_{1} \leq v_{1}$. Then from a mean value theorem, we obtain

$$
f_{1}\left(t, \bar{u}_{1}, \bar{v}_{1}\right)-f_{1}\left(t, u_{1}, v_{1}\right)=\sin \left(\bar{u}_{1}\right)-\sin (u)+\bar{v}_{1}-v_{1} \geq-1\left[u-\bar{u}_{1}\right]-1\left[v-\bar{v}_{1}\right],
$$

thus $L_{1}(t)=1, L_{2}(t)=1$. Let $\bar{e}_{1} \leq e_{1}$ and $\bar{r}_{1} \leq r_{1}$. Then

$$
\begin{aligned}
f_{2}\left(t, \bar{e}_{1}, \bar{r}_{1}\right)-f_{1}\left(t, e_{1}, r_{1}\right) & =\bar{e}_{1} \sin (t)-e_{1} \sin (t)+0.4 \bar{r}_{1} \cos (t)-0.4 r_{1} \cos (t) \\
& =-\sin (t)\left[e_{1}-\bar{e}_{1}\right]-0.4 \cos (t)\left[r_{1}-\bar{r}_{1}\right],
\end{aligned}
$$

thus $L_{3}(t)=-\sin (t), L_{4}(t)=-0.4 \cos (t)$. Thus conditions (7.9) and (7.10) are satisfied. It is easy to show that

$$
\frac{1}{1-0.3} \int_{0}^{1}\left(\int_{0}^{s}[2-\sin (t)-0.4 \cos (t)] d t\right) d s<1
$$

On Figure 7.1, we present approximations of solutions (iterations 6 and 16). Maximal differences between them are

$$
\max _{t \in J}\left|y_{6}(t)-z_{6}(t)\right| \approx 0.035, \quad \max _{t \in J}\left|y_{16}(t)-z_{16}(t)\right| \approx 0.00005
$$




\section{References}

[1] W. Ding, M. Han, and J. Mi, Periodic boundary value problem for the second-order impulsive functional differential equations, Computers \& Mathematics with Applications 50 (2005), no. 34, 491-507.

[2] T. Jankowski, Advanced differential equations with nonlinear boundary conditions, Journal of Mathematical Analysis and Applications 304 (2005), no. 2, 490-503.

[3] _ On delay differential equations with nonlinear boundary conditions, Boundary Value Problems 2005 (2005), no. 2, 201-214.

[4] Solvability of three point boundary value problems for second order differential equations with deviating arguments, Journal of Mathematical Analysis and Applications 312 (2005), no. 2, 620-636.

[5] _ Boundary value problems for first order differential equations of mixed type, Nonlinear Analysis 64 (2006), no. 9, 1984-1997.

[6] D. Jiang, M. Fan, and A. Wan, A monotone method for constructing extremal solutions to secondorder periodic boundary value problems, Journal of Computational and Applied Mathematics 136 (2001), no. 1-2, 189-197.

[7] D. Jiang and J. Wei, Monotone method for first- and second-order periodic boundary value problems and periodic solutions of functional differential equations, Nonlinear Analysis 50 (2002), no. 7, 885-898.

[8] D. Jiang, P. Weng, and X. Li, Periodic boundary value problems for second order differential equations with delay and monotone iterative methods, Dynamics of Continuous, Discrete \& Impulsive Systems. Series A 10 (2003), no. 4, 515-523.

[9] V. Kolmanovskii and A. Myshkis, Introduction to the Theory and Applications of Functional Differential Equations, Mathematics and Its Applications, vol. 463, Kluwer Academic, Dordrecht, 1999.

[10] G. S. Ladde, V. Lakshmikantham, and A. S. Vatsala, Monotone Iterative Techniques for Nonlinear Differential Equations, Monographs, Advanced Texts and Surveys in Pure and Applied Mathematics, vol. 27, Pitman, Massachusetts, 1985.

[11] J. J. Nieto and R. Rodríguez-López, Existence and approximation of solutions for nonlinear functional differential equations with periodic boundary value conditions, Computers \& Mathematics with Applications 40 (2000), no. 4-5, 433-442.

[12] __ Remarks on periodic boundary value problems for functional differential equations, Journal of Computational and Applied Mathematics 158 (2003), no. 2, 339-353.

T. Jankowski: Department of Differential Equations, Gdansk University of Technology, 11/12 G.

Narutowicz Street, 80-952 Gdańsk, Poland

E-mail address: tjank@mifgate.pg.gda.pl

W. Szatanik: Department of Differential Equations, Gdansk University of Technology, 11/12 G.

Narutowicz Street, 80-952 Gdańsk, Poland

E-mail address: wawrzyniec@mifgate.pg.gda.pl 